\title{
Fibre sequences and localization of simplicial sheaves
}

\author{
MatThias WendT
}

\begin{abstract}
In this paper, we discuss the theory of quasifibrations in proper Bousfield localizations of model categories of simplicial sheaves. We provide a construction of fibrewise localization and use this construction to generalize a criterion for locality of fibre sequences due to Berrick and Dror Farjoun. The results allow a better understanding of unstable $\mathbb{A}^{1}$-homotopy theory.
\end{abstract}

55R65, 55P60; 18F20, 14F42

\section{Introduction}

In this paper, we discuss aspects of Bousfield localization for simplicial sheaves. One of the main phenomena of interest is the behaviour of fibrations resp. fibre sequences under a Bousfield localization. In general, fibrations and fibre sequences are not preserved by a Bousfield localization, and it is an interesting question to find suitable criteria under which they are preserved. An extensive discussion of issues related to this question can be found in Dror Farjoun [5]. A general criterion for locality of fibre sequences in nullifications has been obtained by Berrick and Dror Farjoun in [1]. The main goal of this paper is to provide a generalization of this result to the setting of simplicial sheaves. It should be pointed out that the methods heavily use homotopy pullbacks and therefore only apply to the case where the Bousfield localization is right proper.

The main tool used in the present work is an analogue of the theory of quasifibrations of Dold and Thom [3]. On the one hand, quasifibrations behave like fibrations in that point set and homotopy fibres agree - in particular, quasifibrations give rise to fibre sequences and hence long exact homotopy sequences. On the other hand, quasifibrations are much more flexible than fibrations. In the setting of categories of simplicial sheaves, the sharp maps of Rezk [15] provide a replacement for quasifibrations for model categories of simplicial sheaves. This theory has been used by the author in [18] to produce classifying spaces for fibre sequences of simplicial sheaves. In the present paper, we consider the notion of universally $f$-local maps, cf Definition 5.1, in (proper) Bousfield localizations of model categories of simplicial sheaves. This notion as well as the basic assertions in Section 5 are due to Jardine and were suggested to me as a correction to a mistake in an earlier version of this paper. Jardine's definition of universally $f$-local 
maps is equivalent to the definition of sharp maps given by Rezk in [15], and the assertions in Section 5 show that universally $f$-local maps provide a good theory of " $f$-local quasifibrations".

There are two simple reasons why the calculus of universally $f$-local maps works in Bousfield localizations of simplicial sheaves: on the one hand, one can use the homotopy colimit decomposition and homotopy distributivity of simplicial sheaves. On the other hand, the properness of the localized model structure has the important consequence that a simplicial quasifibration over an $f$-local base is an $f$-local quasifibration.

With the $f$-local quasifibrations, it is possible to give a construction of fibrewise $f$-localization. The construction we give in Section 6 is almost a direct translation of the fibrewise localization in the category of simplicial sets-again the main technical tools are the homotopy colimit decomposition and the properness of the local model structure.

Once we have a working construction of fibrewise localization, we can almost directly translate the criterion of Berrick and Dror Farjoun to the simplicial sheaf setting. The result is then the following.

Theorem 7.3 Let $T$ be a site and let $f: X \rightarrow Y$ be a morphism of simplicial sheaves in $\Delta^{\mathrm{op}} \mathcal{S h v}(T)$. Assume that the $f$-local model structure is proper. Let $p: E \rightarrow B$ be a simplicial fibration of simplicial sheaves.

We denote by $\bar{p}: \bar{E} \rightarrow B$ the fibrewise $f$-localization of $p$, and by $j: B \rightarrow L_{f} B$ an $f$-local fibrant replacement of $B$. The following are equivalent, where (iv) only makes sense if $p: E \rightarrow B$ is locally trivial:

(i) the map $p: E \rightarrow B$ is universally $f$-local;

(ii) the fibrewise localization $\bar{p}: \bar{E} \rightarrow B$ is universally $f$-local;

(iii) for each simplex $\sigma: \Delta^{n} \times U \rightarrow L_{f} B$, the following canonical diagram is a simplicial homotopy pullback:

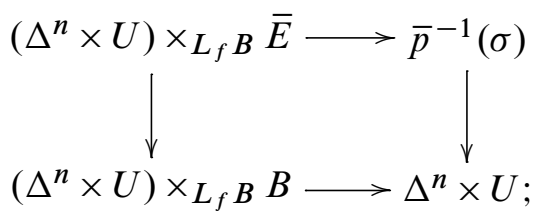

$\bar{p}^{-1}(\sigma)$ denotes the fibre of the fibrewise localization over $\sigma$, cf Definition 6.4; 
(iv) for each simplex $\sigma: \Delta^{n} \times U \rightarrow L_{f} B$, the composition

$$
\left(\Delta^{n} \times U\right) \times_{L_{f} B} B=j^{-1}(\sigma) \rightarrow B \rightarrow B \text { hAut॰ }(F) \rightarrow B \text { hAut॰ }\left(L_{f} F\right)
$$

factors through the projection $\left(\Delta^{n} \times U\right) \times_{L_{f} B} B \rightarrow \Delta^{n} \times U$ (in the simplicial homotopy category).

Note that for the trivial site $T=*$, the above result specializes exactly to [1, Theorem 4.1]. The additional complication in the formulation of the above theorem is due to the fact that the homotopy colimit decomposition of a simplicial sheaf allows to decompose a simplicial sheaf $X$ as the homotopy colimit of its simplices $\Delta^{n} \times U \rightarrow X$, but the spaces $\Delta^{n} \times U$ are not necessarily contractible. However, the interpretation of the above theorem is still the same: a map of simplicial sheaves $p: E \rightarrow B$ is universally $f$-local if the restriction of its fibrewise localization $\bar{p}: \bar{E} \rightarrow B$ to nonlocal parts of $B$ is "trivial". Here nonlocal parts of $B$ are fibres of $j: B \rightarrow L_{f} B$ over simplices $\Delta^{n} \times U \rightarrow L_{f} B$, and "trivial" means that the corresponding map is a pullback of a map over $\Delta^{n} \times U$.

As an interesting application, we arrive at conditions when morphisms induce fibre sequences in $\mathbb{A}^{1}$-homotopy theory. In the case where the morphisms are locally trivial in the Nisnevich topology, the homotopy theory criteria reduce to a simple condition on the sheaf of homotopy self-equivalences of the fibre; see Corollary 8.2.

Theorem 1 Let $F$ be a simplicial sheaf on $\operatorname{Sm}_{k}$. If $\pi_{0}$ hAut. $L_{\mathbb{A}^{1}} F$ is a strongly $\mathbb{A}^{1}$-invariant sheaf of groups, then any morphism $p: E \rightarrow B$ which is locally trivial in the Nisnevich topology with fibre $F$ is universally $\mathbb{A}^{1}$-local. In particular, there are $\mathbb{A}^{1}$-local fibre sequences $F \rightarrow E \rightarrow B$ for any choice of base point of $B$.

Structure of the paper We first discuss the work of Berrick and Dror Farjoun in Section 2. In Section 3, we recall preliminaries on model structures on categories of simplicial sheaves, in particular homotopy distributivity and homotopy colimit decomposition. In Section 4, we recall preliminaries on the Bousfield localization of simplicial sheaves, in particular regarding properness of the localized model structure. Then Section 5 provides an exposition of Jardine's universally $f$-local maps and their properties. These properties are used in Section 6 to construct a fibrewise localization for fibrations of simplicial sheaves. Section 7 provides the main characterization result for universally $f$-local maps which generalizes the result of Berrick and Dror Farjoun. Finally, Section 8 discusses applications to $\mathbb{A}^{1}$-homotopy theory. 
Acknowledgements The results presented here are taken from my $\mathrm{PhD}$ thesis [17] which was supervised by Annette Huber-Klawitter. I would like to use the opportunity to thank her for her encouragement and interest in my work. I would also like to thank Rick Jardine for pointing out a mistake in an earlier version, and for his extremely helpful letter on universally $f$-local maps. All the material in Section 5 is due to Jardine and is included in this paper with his permission. The present paper would not have its present form without his input. Finally, I would like to thank the referees for a careful reading and helpful comments.

\section{The work of Berrick and Dror Farjoun}

In this section, we provide a short review of the original work of Berrick and Dror Farjoun [1] as well as a comparison with our attempt at a generalization to simplicial sheaves. In general, Bousfield localization does not preserve fibre sequences, and it is an interesting and important question to find suitable conditions under which a fibre sequence is preserved by Bousfield localization — several chapters of [5] discuss the ramifications of this question. The result of Berrick and Dror Farjoun [1, Theorem 0.1] gave a very conceptual answer: a fibre sequence $F \rightarrow E \rightarrow B$ is preserved by a nullification if and only if the pullback of the fibrewise localization to the "nonlocal part" of $B$ is trivial. Though still difficult to check, it provides a satisfactory conceptual answer.

There are several building blocks of the result of Berrick and Dror Farjoun: one is a characterization of nullifications as those localizations where fibre sequences with local bases are preserved. This in particular implies that the property of being preserved is stable under pullbacks of fibre sequences. Second, the fibrewise localization provides an intermediary between the original fibre sequence and its localization. With these two major tools in hand, the main theorem is then obtained by comparing a fibre sequence with its fibrewise localization, using suitable homotopy pullback squares.

Our goal in this paper is a generalization of the result of Berrick and Dror Farjoun to model categories of simplicial sheaves. There are several modifications necessary, which we want to outline.

- The two major problems to be addressed are the following: first, in the localization theory for topological spaces, there are frequent connectivity assumptions. However, there are many relevant situations where nonconnected simplicial sheaves appear which can not simply be decomposed into their connected components. The other problem, which makes the formulation of Theorem 7.3 more complicated, is that the simplicial sheaf setting does not allow us to assume that the spaces we are dealing with are locally 
contractible: the objects of the base site provide many different local models from which the spaces are glued.

- Since we do not have connectivity or base point assumptions, the characterization result [1, Theorem 2.1] can not immediately be generalized. We can reformulate some of the properties, though: (ii) and (iii) make sense in any pointed model category and are consequences of properness. Properness of nullifications has been proved by Jardine [12, Appendix A]. Property (iv) can be reformulated (with our definition of fibrewise localization) as "the fibrewise localization of the coaugmentation $X \rightarrow L X$ induces a simplicial weak equivalence $\bar{X} \rightarrow L X$ ”. An analogue of [1, Theorem 2.1] would then state that an $f$-localization of a model category of simplicial sheaves is proper if and only if it is Quillen equivalent to a nullification. Of course, one half is true by Jardine's result, but it is not clear to me if the converse is true. The proof of the implication (iv) $\Rightarrow$ (i) in [1] can not be applied, simply because it uses connectivity assumptions and it is not obvious what the suitable replacement of $A_{L} U \vee A_{L} V$ should be.

- The way around connectivity and local contractibility is provided by universally $f$-local maps. These are maps which by definition have the right $f$-local homotopy fibres. Therefore, they are the appropriate replacement of fibre sequences preserved by the localization. From properness, it is then possible to deduce pullback stability, without having an analogue of [1, Theorem 2.1]. This is discussed in Section 5.

- A central tool used in [1] is the fibrewise localization. Again, its usual definition via the simplex category makes use of a local contractibility hypothesis. A slightly more complicated definition taking into account the different objects of the site is provided in Section 6.

- Having a replacement for pullback stability and fibrewise localization, the argument of Berrick and Dror Farjoun can be translated almost verbatim to the setting of simplicial sheaves. The formulation of the theorem changes for two reasons. On the one hand, the characterization via the classifying spaces of fibrations do not generally work, again for lack of local contractibility. It can only be applied when the map in question is locally trivial. Also, the space $A_{L} B$ from [1] can not be used, for lack of connectivity. We use instead a pullback square which in the special case of simplicial sets reduces exactly to the triviality of $L F \rightarrow E_{1} \rightarrow A_{L} B$. 


\section{Preliminaries on simplicial sheaves}

\subsection{Model structures on simplicial sheaves}

We will be working in categories of simplicial sheaves. The underlying site is usually denoted by $T$, the category of sheaves on it by $\operatorname{Shv}(T)$, and the category of simplicial sheaves by $\Delta^{\text {op }} \mathcal{S} h v(T)$. On this category, there are several model structures all yielding the same homotopy theory. We will use the injective model structure, cf Jardine [11, Theorems 18 and 27].

Theorem 3.1 Let $\mathcal{E}$ be a topos. Then the category $\Delta^{\mathrm{op}} \mathcal{E}$ of simplicial objects in $\mathcal{E}$ has a model structure, where the

(i) cofibrations are monomorphisms,

(ii) weak equivalences are detected on a fixed Boolean localization,

(iii) fibrations are determined by the right lifting property.

Moreover, the above definition of weak equivalences does not depend on the Boolean localization.

The following proposition recalls the basic properties of this model structure. Existence is proved by Jardine in [11, Theorems 18 and 27]. Properness and simpliciality are proved in [11, Theorem 24]. Cellularity is proved by Hornbostel in [9, Theorem 1.4].

Proposition 3.2 Let $T$ be any Grothendieck site. Then the injective model structure of Jardine on the category of (pre)sheaves of simplicial sets on $T$ is a proper simplicial and cellular model structure.

\subsection{Homotopy pullbacks}

Recall that a commutative square in a model category $\mathcal{C}$,

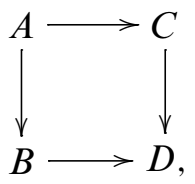

is called a homotopy pullback if for some factorization $C \rightarrow \widetilde{C} \rightarrow D$ as a trivial cofibration $C \rightarrow \widetilde{C}$ and a fibration $\widetilde{C} \rightarrow D$, the induced map $A \rightarrow B \times{ }_{D} \widetilde{C}$ is a weak equivalence. This notion is only well-defined if the model category $\mathcal{C}$ is proper, $\mathrm{cf}$ 
Goerss and Jardine [7, Section II.8]. As homotopy pullbacks play a major role in this paper, all the model categories in sight will be assumed to be proper.

An important special case of homotopy pullbacks are those of the form

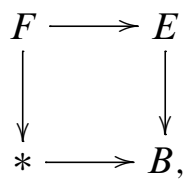

ie in which one of the factors is contractible. Such pullbacks are basically the same thing as fibre sequences. As there is always a problem with base points and different homotopy types of simplices in categories of sheaves, it is better to talk generally about homotopy pullbacks rather than fibre sequences.

\subsection{Homotopy distributivity and colimit decomposition}

Next, we repeat several basic statements on the behaviour of homotopy limits and colimits in categories of simplicial sheaves. The main result needed is the homotopy distributivity of Rezk, cf [15]. Results and preliminaries can also be found by the author in [18, Section 2].

Recall that a diagram $\mathcal{X}: \mathcal{I} \rightarrow \mathcal{C}$ in a model category $\mathcal{C}$ is called homotopy colimit diagram if the canonical map hocolim $\mathcal{X} \rightarrow \operatorname{colim} \mathcal{X}$ is a weak equivalence. We can now recall the definition of homotopy distributivity: let $\mathcal{C}$ be a simplicial model category, let $\mathcal{I}$ be a small category, and let $f: \mathcal{X} \rightarrow \mathcal{Y}$ be a morphism of $\mathcal{I}$-diagrams in $\mathcal{C}$. The diagrams we are most interested in are the following.

For any $i \in \mathcal{I}$, we have a commutative square

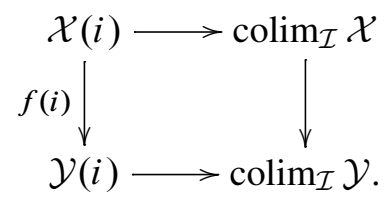

Moreover, for any $\alpha: i \rightarrow j$ in $\mathcal{I}$ we have a commutative square

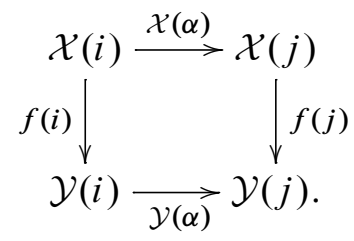


Definition 3.3 (Homotopy Distributivity) In the above situation, we say that $\mathcal{C}$ satisfies homotopy distributivity if for any morphism $f: \mathcal{X} \rightarrow \mathcal{Y}$ of $\mathcal{I}$-diagrams in $\mathcal{C}$ for which $\mathcal{Y}$ is a homotopy colimit diagram, ie $\operatorname{hocolim}_{\mathcal{I}} \mathcal{Y} \rightarrow \operatorname{colim}_{\mathcal{I}} \mathcal{Y}$ is a weak equivalence, the following two properties hold:

(HDi) if each square of the form (1) is a homotopy pullback, then $\mathcal{X}$ is a homotopy colimit diagram;

(HDii) if $\mathcal{X}$ is a homotopy colimit diagram, and each diagram of the form (2) is a homotopy pullback, then each diagram of the form (1) is also a homotopy pullback.

Proposition 3.4 Let $T$ be a site. Then homotopy distributivity holds in the model category $\Delta^{\mathrm{op}} \mathcal{S} h v(T)$.

The main consequence of homotopy distributivity is the canonical homotopy colimit decomposition of morphisms of simplicial sheaves. This allows to write the source of a morphism as homotopy colimit of its fibres over simplices of the target. We first recall the homotopy colimit decomposition for simplicial sets: for a simplicial set $X$, we can consider its category of simplices $\boldsymbol{\Delta} \downarrow X$ whose objects are morphisms $\Delta^{n} \rightarrow X$ and whose morphisms are the obvious commutative triangles. The notation $\Delta \downarrow X$ is chosen because the category of simplices is the comma category of objects under the standard simplices. For a morphism of simplicial sets $f: X \rightarrow Y$, one can then associate a functor $f^{-1}: \Delta \downarrow Y \rightarrow \Delta^{\text {op }} \mathcal{S}$ et by mapping a simplex $\sigma: \Delta^{n} \rightarrow Y$ to the simplicial set $f^{-1}(\sigma)$ defined by the following pullback diagram:

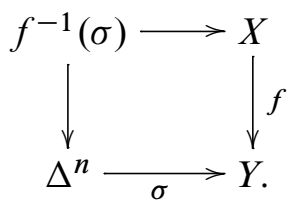

There is a canonical morphism of simplicial sets hocolim $f^{-1} \rightarrow X$ which is a weak equivalence, cf Goerss and Jardine [6, Lemma IV.5.2]. A similar statement holds for simplicial sheaves. The right notion to formulate it is the canonical homotopy colimit decomposition for objects in a combinatorial model category, cf Dugger [4]. For the convenience of the reader we recall notation and (a generalization of) a lemma already formulated in [18, Section 2.8]. Let $\mathcal{C}$ be a combinatorial model category, $\mathcal{T}$ be a small category. For any functor $I: \mathcal{T} \rightarrow \mathcal{C}$ and a fixed cosimplicial resolution $\Gamma_{I}: \mathcal{T} \rightarrow \Delta \mathcal{C}$, we obtain a functor $\mathcal{T} \times \boldsymbol{\Delta} \rightarrow \mathcal{C}:(U,[n]) \mapsto \Gamma(n)(U)$ which replaces the standard cosimplicial object $\boldsymbol{\Delta}$ in $\Delta^{\text {op }} \mathcal{S}$ et above. For any object $X$, we can consider the overcategory $(\mathcal{T} \times \boldsymbol{\Delta} \downarrow X)$ and the canonical diagram $(\mathcal{T} \times \boldsymbol{\Delta} \downarrow X) \rightarrow \mathcal{C}: \Gamma(n)(U) \mapsto U \times \Delta^{n}$ 
which is the proper replacement for the category of simplices. We mostly deal with the special case where $\mathcal{C}=\Delta^{\mathrm{op}} \mathcal{S} h v(T)$ is the model category of simplicial sheaves on a site $T, \mathcal{T}=T$ is chosen to be the site, and the cosimplicial resolution is induced from the standard cosimplicial object $\Delta$ in $\Delta^{\text {op }} \mathcal{S} h v(T)$. In this case, the category $(\mathcal{T} \times \boldsymbol{\Delta} \downarrow X)$ is the "usual" category of simplices whose objects are the simplices $X(U)_{n}$ of $X$ over $U$, and morphisms are the appropriate commutative diagrams.

The following lemma was formulated in [18] only for a fibration of fibrant simplicial sheaves. In this special case, its proof is an application of homotopy distributivity.

Lemma 3.5 Let $T$ be a site, and let $p: E \rightarrow B$ be a morphism of simplicial sheaves. Then $p$ is weakly equivalent to the morphism of simplicial sheaves

$$
\operatorname{hocolim} \mathcal{F} \rightarrow \operatorname{hocolim}(T \times \Delta \downarrow B),
$$

where $(T \times \boldsymbol{\Delta} \downarrow B)$ is the canonical diagram associated to some fixed cosimplicial resolution, and the diagram $\mathcal{F}$ is the diagram of fibres:

$$
\mathcal{F}:(T \times \Delta \downarrow B) \rightarrow \Delta^{\mathrm{op}} \mathcal{S h v}(T):\left(U \times \Delta^{n} \rightarrow B\right) \mapsto\left(U \times \Delta^{n}\right) \times{ }_{B} E .
$$

Proof We have a composition of morphisms

$$
\operatorname{hocolim} \mathcal{F} \rightarrow \operatorname{colim} \mathcal{F} \rightarrow E .
$$

The second morphism is an isomorphism by the distributivity in categories of sheaves, cf eg [15, Proposition 3.7]. It then suffices to prove that the diagram $\mathcal{F}$ is a homotopy colimit diagram.

If the topos has enough points, this can be checked on points, cf [18, Corollary 2.10]. For a point $x$ of the topos $\operatorname{Sh} v(T)$, the diagram $x^{*}(\mathcal{F})$ is the diagram of fibres of the simplicial set map $x^{*}(p): x^{*}(E) \rightarrow x^{*}(B)$ :

$$
\begin{gathered}
x^{*}(\mathcal{F}):\left(\Delta \downarrow x^{*}(B)\right) \rightarrow \Delta^{\mathrm{op}} \mathcal{S e t}: \\
\left(\sigma: \Delta^{n} \rightarrow x^{*}(B)\right) \mapsto\left(x^{*}(p)\right)^{-1}(\sigma)=\Delta^{n} \times_{x^{*}(B)} x^{*}(E) .
\end{gathered}
$$

In particular, the composition $x^{*}($ hocolim $\mathcal{F}) \rightarrow x^{*}(\operatorname{colim} \mathcal{F}) \rightarrow E$ is the composition $\operatorname{hocolim}\left(x^{*}(\mathcal{F})\right) \rightarrow \operatorname{colim}\left(x^{*}(\mathcal{F})\right) \rightarrow x^{*}(E)$. But the latter is known to be a homotopy colimit diagram, cf [7, IV.5.2].

The same argument as above shows that the assertion is true in the presheaf category, because colimits (and therefore homotopy colimits) of simplicial presheaves are computed pointwise. The general case then follows from the properties of the sheafification functor. 
Corollary 3.6 Consider the following commutative triangle, in which $p_{1}$ and $p_{2}$ are fibrations:

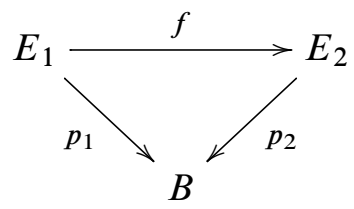

Then the morphism $f$ is a weak equivalence if one of the following holds:

(i) the induced morphisms $p_{1}^{-1}(\sigma) \rightarrow p_{2}^{-1}(\sigma)$ are weak equivalences for all objects $\sigma: \Delta^{n} \times U \rightarrow B$ of the category of simplices $(\Delta \times T) \downarrow B$;

(ii) the induced morphisms $p_{1}^{-1}(x) \rightarrow p_{2}^{-1}(x)$ are weak equivalences for all maps $x: U \rightarrow B$.

Proof (i) We have a commutative square

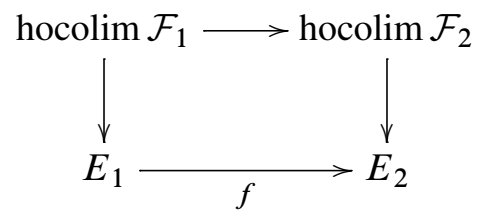

in which the two vertical morphisms are weak equivalences by Lemma 3.5. The top horizontal morphism is a homotopy colimit of weak equivalences, therefore the bottom horizontal morphism is a weak equivalence.

Part (ii) follows from (i) by considering the following diagram in which all squares are homotopy pullbacks:

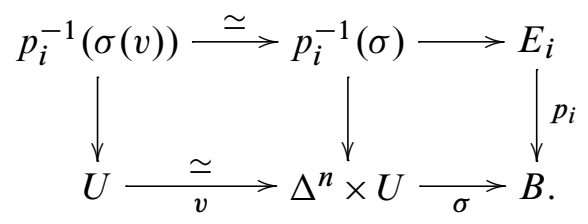

The right square is a homotopy pullback because $p_{i}$ is a fibration (and the model structure is proper), the left because there are two parallel weak equivalences. It then suffices to check weak equivalences after restriction to vertices of simplices. 


\section{Preliminaries on localization functors}

\subsection{Bousfield Localization}

We repeat the standard definitions of local objects and local weak equivalences. These definitions can be found in [5] and Hirschhorn [8] for the case of simplicial sets, and in Morel and Voevodsky [14] for the case of simplicial sheaves.

Let $\mathcal{C}$ be a model category, and let $f: X \rightarrow Y$ be a morphism of cofibrant objects.

Definition 4.1 (Local objects, weak equivalences) An object $A \in \mathcal{C}$ is called $f$-local if $A$ is fibrant and the following morphism is a bijection for each $B \in \mathrm{Ho} \mathcal{C}$ :

$$
\operatorname{hom}_{\mathrm{Ho}} \mathcal{C}(B \times Y, A) \rightarrow \operatorname{hom}_{\mathrm{Ho}} \mathcal{C}(B \times X, A) \text {. }
$$

A morphism $g: A \rightarrow B \in \mathcal{C}$ is called an $f$-local weak equivalence if for any $f$-local object $C$, the following morphism is a bijection:

$$
\operatorname{hom}_{\mathrm{Ho}} \mathcal{C}(B, C) \rightarrow \operatorname{hom}_{\mathrm{Ho}} \mathcal{C}(A, C) .
$$

Remark 4.2 (i) The above is the definition of local given in [14]. It is easy to check that it coincides with the definition in [6], where one requires a weak equivalence of simplicial sets:

$$
\operatorname{Hom}(B, C) \rightarrow \operatorname{Hom}(A, C) .
$$

This in turn is equivalent to requiring weak equivalences on internal homomorphisms:

$$
\operatorname{Hom}(B, C) \rightarrow \operatorname{Hom}(A, C) .
$$

(ii) Note that there is a difference between pointed and unpointed. The definition above is for a general model category, using unpointed mapping spaces. In a pointed model category, one uses the pointed mapping spaces. For connected objects both notions coincide.

Of course, one can consider more general localizations, ie localizations with respect to a set of maps as in [14, Section 2.2], or homology localization as in [6, Section 3]. If $f$ is nullhomotopic such a localization is also called nullification, and we also use $L_{W}$ to denote the corresponding localization functor. The most important applications we have in mind are the $\mathbb{A}^{1}$-nullification functors $L_{\mathbb{A}^{1}}$ on $\Delta^{\mathrm{op}} \mathcal{S} h v\left(\operatorname{Sm}_{S}\right)$. 


\subsection{Localization functors}

This paragraph repeats the theorem on existence and universality of localization functors for simplicial sheaves. Most of the elementary facts in [5, 1.A.8] are easy consequences of this theorem, which is proved in [14, Theorem 2.2.5] and in similar form in Goerss and Jardine [6, Theorem 4.4].

We start recalling necessary definitions related to localization functors in a general model category.

Definition 4.3 A functor $F: \mathcal{C} \rightarrow \mathcal{C}$ is called coaugmented if there is a natural transformation $j: \operatorname{id}_{\mathcal{C}} \rightarrow F$. A coaugmented functor $F$ is called homotopically idempotent if the two natural maps $j_{F A}, F j_{A}: F A \rightrightarrows F F A$ are weak equivalences and homotopic to each other. The coaugmentation map $j_{A}$ is homotopy universal with respect to maps into local spaces if any map $A \rightarrow B$ into a local space $T$ factors uniquely (up to homotopy) through $j_{A}: A \rightarrow F A$. The functor $F$ is called simplicial if it is compatible with the simplicial structure, ie if there exist functorial morphisms $\sigma:(F A) \otimes K \rightarrow F(A \otimes K)$ for any object $A \in \mathcal{C}$ and any simplicial set $K$. These morphisms have to satisfy some rather obvious conditions described in [5, Definition 1.C.8]. The functor $F$ is called continuous if it induces a morphism on inner function spaces

$$
\operatorname{Hom}(A, B) \rightarrow \operatorname{Hom}(F A, F B),
$$

which is compatible with composition.

We recall the existence of localizations for simplicial sheaf categories from [6, Theorem 4.4], which is the proper generalization of [5, Theorem A.3]. The existence of the $f$-local model structure is proven in [6, Theorem 4.8]. Note that the existence of localizations for simplicial sheaves is a global result, in the sense that it does not simply follow from the existence of localizations of simplicial sets by looking at the points of the topos.

Theorem 4.4 Let $f: X \rightarrow Y$ be any cofibration in $\Delta^{\text {op }} \mathcal{S h v}(T)$ and suppose $\alpha$ is an infinite cardinal which is an upper bound for the cardinalities of both $Y$ and the set of morphisms of $T$. Then there exists a functor $L_{f}$, called the $f$-localization functor, which is coaugmented and homotopically idempotent. Any two such functors are naturally weakly equivalent to each other. The map $A \rightarrow L_{f} A$ is a homotopically universal map to $f$-local spaces. Moreover, $L_{f}$ can be chosen to be simplicial and continuous.

There is a simplicial model structure on $\Delta^{\mathrm{op}} \mathcal{S h v}(T)$ where the cofibrations are monomorphisms, weak equivalences are $f$-local weak equivalences and fibrations are defined via the right lifting property. 


\subsection{Properness}

In [8, Chapter 3], Bousfield localizations of general model categories are investigated. As shown in [8, Proposition 3.4.4 and Theorem 4.1.1], left Bousfield localizations preserve left properness, ie the left Bousfield localization of a left proper model category is again left proper.

The $f$-local model structure for a morphism $f: X \rightarrow Y$ is not in general right proper. It is known [12, Theorem A.5], that the $f$-local model structure is proper if $f$ is of the form $* \rightarrow I$, ie $L_{f}$ is a nullification. A special case of this is the properness of the homotopy theory of a site with interval, which is proved in [14, Theorem 2.2.7 and Section 2.3].

We mention again that we will be working a lot with $f$-local homotopy pullbacks, ie homotopy pullbacks in the $f$-local model structure. Therefore, throughout the rest of the paper, we will assume that $f: X \rightarrow Y$ is a morphism of simplicial sheaves on a site $T$ such that the $f$-local model structure on $\Delta^{\text {op }} \mathcal{S h v}(T)$ is proper. Most of the time, this will be explicitly mentioned anyway.

\section{$5 f$-local sharp maps: universally $f$-local maps}

In this section, we will discuss a class of maps called universally $f$-local maps, which should be thought of as " $f$-local quasifibrations"-they are not necessarily fibrations in the $f$-local model structure but give rise to $f$-local fibre sequences.

Definition 5.1 Let $T$ be a site and let $f: X \rightarrow Y$ be a morphism of simplicial sheaves in $\Delta^{\mathrm{op}} \mathcal{S h v}(T)$ such that the $f$-local model structure is proper. A morphism $p: E \rightarrow B$ of simplicial sheaves is called universally $f$-local if for any representable $U \in T$ and any simplex $\sigma: \Delta^{n} \times U \rightarrow B$ the following pullback diagram is an $f$-local homotopy pullback:

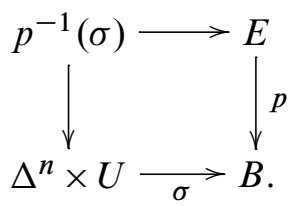

Remark 5.2 (i) As $\sigma: \Delta^{n} \times U \rightarrow B$ ranges over the various simplices of the base simplicial sheaf $B, p^{-1}(\sigma)$ ranges through the possible fibres of $p: E \rightarrow B$. The definition of universally $f$-local map makes sure that all these objectswhich could be called local homotopy fibres of $p$-have the right homotopy type. Note however, that the different representable objects $U \in T$ usually have 
different homotopy types in $\Delta^{\text {op }} \mathcal{S h v}(T)$, so that for different $U_{1}, U_{2}$, the fibres over $\Delta^{n} \times U_{1} \rightarrow B$ and $\Delta^{m} \times U_{2} \rightarrow B$ will usually not be weakly equivalentthis is the major difference to the case of simplicial sets where all simplices $\Delta^{n}$ are weakly equivalent to the point.

(ii) Note that if $\mathcal{C}$ is a model category with a terminal object pt, $f: X \rightarrow Y$ is a morphism in $\mathcal{C}, p: E \rightarrow B$ is universally $f$-local and $x: \mathrm{pt} \rightarrow E$ is a choice of basepoint of $E$, then $\left(p^{-1}(p(x)), x\right) \rightarrow(E, x) \rightarrow(B, p(x))$ is a fibre sequence in the sense of Hovey [10, Definition 6.2.6] in the pointed model category $(\mathcal{C}, \mathrm{pt})$. In particular, a universally $f$-local map induces long exact homotopy sequences for any choice of base points.

Even more is true: any pullback involving a universally $f$-local map is an $f$-local homotopy pullback, provided of course the respective $f$-local model structure is proper.

Lemma 5.3 Let $T$ and $f: X \rightarrow Y$ be as in Definition 5.1, and assume that the $f$-localization of $\Delta^{\mathrm{op}} \mathcal{S h v}(T)$ is proper. A map $p: E \rightarrow B$ of simplicial sheaves is universally $f$-local if and only if for all morphisms $g: Z \rightarrow B$ the following pullback diagram is an $f$-local homotopy pullback:

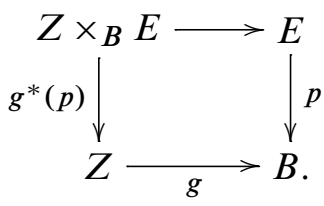

Proof The "if"-direction is clear, so let $p: E \rightarrow B$ be universally $f$-local. Factor $p$ as

$$
p: E \stackrel{j}{\longrightarrow} \widetilde{E} \stackrel{q}{\longrightarrow} B,
$$

where $j: E \rightarrow \widetilde{E}$ is an $f$-local weak equivalence and $q: \widetilde{E} \rightarrow B$ is an $f$-local fibration. Using properness of the $f$-local model structure, we need to show that the induced map $Z \times_{B} E \rightarrow Z \times_{B} \widetilde{E}$ is an $f$-local weak equivalence. By Lemma 3.5, this map is weakly equivalent to the map of homotopy colimits

$$
\underset{T \times \boldsymbol{\Delta} \downarrow Z}{\operatorname{hocolim}}\left(g^{*}(p)\right)^{-1}(\sigma) \rightarrow \underset{T \times \boldsymbol{\Delta} \downarrow Z}{\operatorname{hocolim}}\left(g^{*}(q)\right)^{-1}(\sigma),
$$

where the diagram $\left(g^{*}(p)\right)^{-1}(\sigma)$ is the diagram of the fibres of $g^{*}(p): Z \times{ }_{B} E \rightarrow Z$ over the simplices $\sigma: \Delta^{n} \times U \rightarrow Z$ of $Z$, and the same for $\left(g^{*}(q)\right)^{-1}(\sigma)$. But for any simplex $\sigma: \Delta^{n} \times U \rightarrow Z$ of $Z$, the induced map

$$
\left(g^{*}(p)\right)^{-1}(\sigma)=p^{-1}(g \circ \sigma) \rightarrow q^{-1}(g \circ \sigma)=\left(g^{*}(q)\right)^{-1}(\sigma)
$$


is an $f$-local weak equivalence, since $p$ was assumed to be universally $f$-local and the $f$-local model structure was assumed to be proper. But then the morphism between diagrams consists of $f$-local weak equivalences only, so the above homotopy colimit is an $f$-local weak equivalence. This shows the claim.

Note that the above result also establishes that the property of being universally $f$-local is stable under pullbacks.

Corollary 5.4 Under the assumptions of Lemma 5.3, if $p: E \rightarrow B$ is universally $f$-local and $g: Z \rightarrow B$ is any morphism of simplicial sheaves, then we have that the map $g^{*}(p): Z \times{ }_{B} E \rightarrow Z$ is universally $f$-local.

The universally $f$-local maps play the role in the $f$-local model category of the quasifibrations in [3], the sharp maps in [15] and the locally trivial morphisms in [18] they are a replacement for honest fibrations that still give rise to fibre sequences but are easier to handle.

Lemma 5.5 Let $T$ and $f: X \rightarrow Y$ be as in Definition 5.1, and assume that the $f$-localization of $\Delta^{\mathrm{op}} \mathcal{S h} v(T)$ is proper. A map $p: E \rightarrow B$ of simplicial sheaves is universally $f$-local if and only if for any diagram

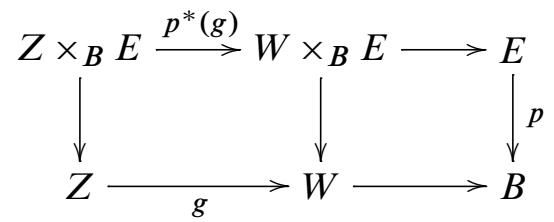

with $g$ an $f$-local weak equivalence, the map $p^{*}(g)$ is also an $f$-local weak equivalence.

Proof If $p$ is universally $f$-local, then the outer square and the right square are $f$-local homotopy pullbacks by Lemma 5.3. By the homotopy pullback lemma (see eg [7, Lemma II.8.22]), the left square is also an $f$-local homotopy pullback. But then it is easy to see that $p^{*}(g)$ must be an $f$-local weak equivalence as well.

Now assume that the condition is satisfied. By Lemma 5.3, it suffices to check that the diagram

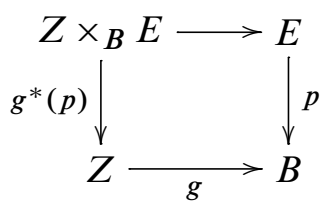


is a homotopy pullback for any map $g: Z \rightarrow B$. Factor $g$ as

$$
g: Z \stackrel{j}{\longrightarrow} W \stackrel{q}{\longrightarrow} B,
$$

where $j: Z \rightarrow W$ is an $f$-local weak equivalence and $q: W \rightarrow B$ is an $f$-local fibration. By the assumption, the morphism $p^{*}(j): Z \times_{B} E \rightarrow W \times_{B} E$ is an $f$-local weak equivalence. Therefore, the above diagram is in fact an $f$-local homotopy pullback, so $p: E \rightarrow B$ is universally $f$-local.

The above result states that universally $f$-local maps are exactly the sharp maps in the sense of Rezk for the $f$-local model structure, cf [15]. It implies in particular that $f$-local fibrations are universally $f$-local. Moreover, it implies that simplicial fibrations over $f$-local bases are universally $f$-local.

Corollary 5.6 Under the assumptions of Lemma 5.5, if $p: E \rightarrow B$ is a simplicial fibration and $B$ is $f$-local and fibrant, then $p$ is universally $f$-local.

Proof This is a consequence of Lemma 5.5 and [12, Lemma A.3].

Our goal in the sequel will be to characterize universally $f$-local maps.

Theorem 5.7 Let $T$ be a site and let $f: X \rightarrow Y$ be a morphism of simplicial sheaves on $\Delta^{\mathrm{op}} \mathcal{S h v}(T)$. Assume that the $f$-local model structure on $\Delta^{\mathrm{op}} \mathcal{S h v}(T)$ is proper.

Let $p: E \rightarrow B$ be a simplicial fibration and let $j: B \rightarrow L_{f} B$ be an $f$-local fibrant replacement. Then $p$ is universally $f$-local if and only if for any $U \in T$ and any simplex $\sigma: \Delta^{n} \times U \rightarrow B$, the induced morphism $p^{-1}(\sigma) \rightarrow(j \circ p)^{-1}(j \circ \sigma)$ is an $f$-local weak equivalence.

Proof Without loss of generality we can assume that $p$ and $j$ are simplicial fibrations: first factor $j$ as $B \rightarrow \widetilde{B} \rightarrow L_{f} B$ with the first map a trivial cofibration and the second map a fibration. Then factor the composition $E \rightarrow B \rightarrow \widetilde{B}$ as $E \rightarrow \widetilde{E} \rightarrow \widetilde{B}$ with the first map a trivial cofibration and the second map a fibration. All the factorizations are done in the simplicial model structure, therefore the replacement $\widetilde{p}: \widetilde{E} \rightarrow \widetilde{B}$ is universally $f$-local if and only if $p: E \rightarrow B$ is.

In the following, we assume that $p$ and $j$ are simplicial fibrations. We want to show that for each $U \in T$ and each simplex $\sigma: \Delta^{n} \times U \rightarrow B$ of $B$, the pullback diagram

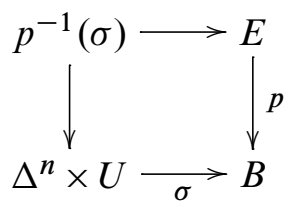


is an $f$-local homotopy pullback. Consider now the diagram

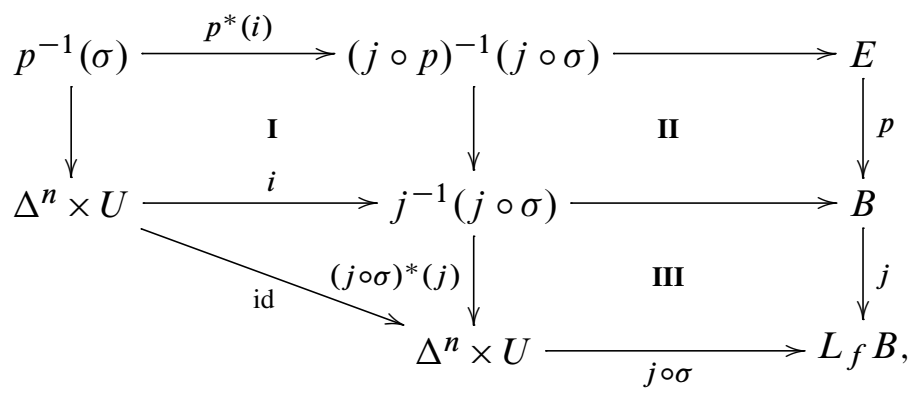

in which the squares I-III are pullbacks. The morphism $i: \Delta^{n} \times U \rightarrow j^{-1}(j \circ \sigma)$ is a consequence of the universal property for the pullback square III.

The squares III and II+III are $f$-local homotopy pullbacks by Corollary 5.6. By the homotopy pullback lemma [7, Lemma II.8.22], the square II is also an $f$-local homotopy pullback. By the same lemma, the square $\mathbf{I}+\mathbf{I I}$ is an $f$-local homotopy pullback if and only if $\mathbf{I}$ is an $f$-local homotopy pullback.

The map $(j \circ \sigma)^{*}(j)$ is an $f$-local weak equivalence since III is a homotopy pullback and $j$ is an $f$-local weak equivalence-it is (a simplicial replacement of) the localization morphism $B \rightarrow L_{f} B$. By 2 out of 3, the map $i: \Delta^{n} \times U \rightarrow j^{-1}(j \circ \sigma)$ is an $f$-local weak equivalence. But then the square $\mathbf{I}$ is an $f$-local homotopy pullback if and only if $p^{*}(i): p^{-1}(\sigma) \rightarrow(j \circ p)^{-1}(j \circ \sigma)$ is an $f$-local weak equivalence. The criterion is proved.

Corollary 5.8 Let $T$ be a site and let $f: X \rightarrow Y$ be a morphism of simplicial sheaves on $\Delta^{\text {op }} \mathcal{S h v}(T)$. Assume that the $f$-local model structure on $\Delta^{\mathrm{op}} \mathcal{S} h v(T)$ is proper.

A morphism $p: E \rightarrow B$ of simplicial sheaves is universally $f$-local if for any diagram

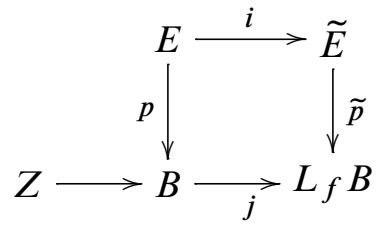

with $j: B \rightarrow L_{f} B$ an $f$-local fibrant replacement, $\tilde{p}$ a simplicial fibration and $i$ a simplicial trivial cofibration, the induced map $Z \times_{B} E \rightarrow Z \times_{L_{f} B} \tilde{E}$ is an $f$-local weak equivalence. 
Proof We complete the diagram in the statement:

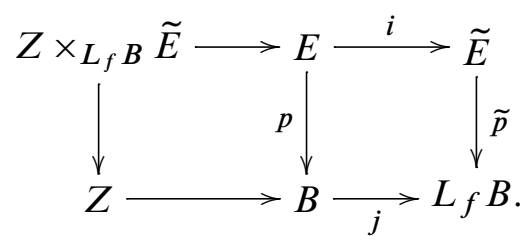

Both the right and the outer square are $f$-local homotopy pullbacks by Corollary 5.6. Consider now the diagram

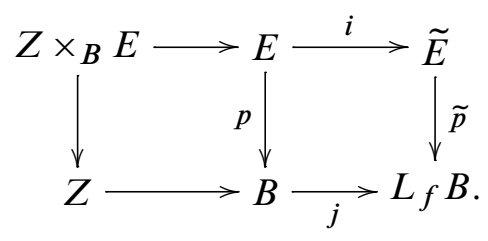

Then $p$ is universally $f$-local if and only if (in every possible such situation) the left square of this diagram is an $f$-local homotopy pullback. But since the right square is an $f$-local homotopy pullback, this left square is an $f$-local homotopy pullback if and only if the outer square is. But because of the previous diagram, the outer square is an $f$-local homotopy pullback if and only if the induced map $Z \times_{B} E \rightarrow Z \times_{L_{f} B} \tilde{E}$ is an $f$-local weak equivalence.

\section{Fibrewise localization}

In this section, we recall several possible definitions of fibrewise localization in categories of simplicial sheaves. For a discussion of fibrewise localization in the category of topological spaces resp. simplicial sets cf [5, Section 1.F] resp. [8, Chapter 6]. For simplicial sets, one can define fibrewise localization as follows. We formulate it for a general localization functor $L$, but we will only be interested in the case where $L=L_{f}$ is localization at a morphism $f: X \rightarrow Y$.

Definition 6.1 Let $L$ be a localization functor on $\Delta^{\text {op }} \mathcal{S}$ et. Then $L$ admits a fibrewise version if for any fibration $p: E \rightarrow B$ of simplicial sets there exists a commutative diagram

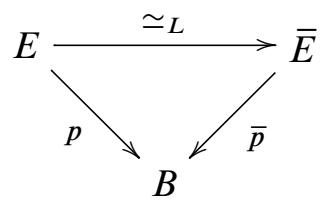


where $\bar{p}$ is a fibration and $E \rightarrow \bar{E}$ a local weak equivalence, such that for each simplex $\sigma: \Delta^{n} \rightarrow B$ the induced morphism $p^{-1}(\sigma) \rightarrow \bar{p}^{-1}(\sigma)$ is (simplicially equivalent to) the localization morphism $p^{-1}(\sigma) \rightarrow L\left(p^{-1}(\sigma)\right)$.

Remark 6.2 We want to note that pointed and unpointed simplicial sets behave rather differently with respect to fibrewise localization. For unpointed simplicial sets, one can construct fibrewise localizations in various different ways, whereas for pointed simplicial sets, one always has to make special connectivity assumptions on the base resp. the fibre because usually there is no continuous choice of base point in a nontrivial fibre sequence $F \rightarrow E \stackrel{p}{\rightarrow} B$. This difference between the unpointed and the pointed setting is also illustrated by [8, Proposition 6.1.4]; see also the discussion in [5, Remark 1.A.7].

The right translation of this to a simplicial sheaf setting is not exactly immediate: the above definition hinges on the fact that $B$ is the homotopy colimit of its simplices, hence a homotopy colimit of contractible spaces. This is no longer true in the simplicial sheaf setting, where $B$ is the homotopy colimit of simplices $\Delta^{n} \times U$ but $U$ is typically not contractible. In the following, we review possible definitions and extensions of fibrewise localization to simplicial sheaves.

\subsection{Fibrewise localization after Chataur and Scherer}

Chataur and Scherer have provided a version of fibrewise localization for general pointed model categories satisfying some axioms, cf Chataur and Scherer [2, Theorem 4.3].

Theorem 6.3 Let $\mathcal{M}$ be a model category which is pointed, left proper, cellular and in which the cube axiom and the ladder axiom holds. Let $L_{f}: \mathcal{M} \rightarrow \mathcal{M}$ be a localization functor which preserves products, and let $p: F \rightarrow E \rightarrow B$ be a fibre sequence in $\mathcal{M}$. Then there exists a fibrewise $f$-localization of $p$.

We note that localization functors of simplicial sheaves commute with finite products as remarked in the proof of [14, Lemma 2.2.32], and that cube and ladder axiom for categories of simplicial sheaves are consequences of homotopy distributivity, $\mathrm{cf}$ Section 3. Therefore, the fibrewise localization method of Chataur and Scherer works in model categories of simplicial sheaves. A result similar to the above can be formulated for fibre sequences over simply connected base spaces, replacing the product condition on $L_{f}$ by the join axiom, cf [2].

Note that the construction of Chataur and Scherer only localizes fibres over the base point. Therefore, this construction of fibrewise localization is not able to deal with the full generality of simplicial sheaves. 


\subsection{Fibrewise localization via classifying spaces}

For a locally trivial morphism $f: E \rightarrow B$ of topological spaces with fibre $F$, one can explain quite easily how to construct the fibrewise localization: Take a trivialization of $f$, ie a covering $U_{i}$ of $X$ over which $\left.f\right|_{U_{i}}: E \times_{B} U_{i} \cong U_{i} \times F \rightarrow U_{i}$. Then apply the simplicial coaugmented functor: on the level of the trivialization one simply replaces the space $F$ by the space $L F$. On the level of transition morphisms, one applies the functor $L$ to the transition map. For this to work we need the functor $L$ to be continuous. This produces an explicit recipe to construct an $L F-$ bundle over $B$.

The same argument can be applied to locally trivial morphisms of simplicial sheaves on a site $T$, in the sense of [18, Definition 3.5]. One can then do the above argument, or use the existence of classifying spaces for locally trivial morphisms as in [18]: if the fibre sequence $F \rightarrow E \rightarrow B$ is locally trivial, it is classified by a morphism $B \rightarrow B$ hAut. $(F)$. Composing with the morphism of classifying space induced from the coaugmentation, we obtain a morphism $B \rightarrow B$ hAut॰ $(F) \rightarrow B$ hAut॰ $(L F)$. Pulling back the universal $L F$-fibre sequence along this morphism produces an $L F$-fibre sequence $L F \rightarrow \bar{E} \rightarrow B$ over $B$, which is the fibrewise localization of the fibre sequence we started with. This implies that in the above situation any locally trivial $F$-fibre sequence of simplicial sheaves $F \rightarrow E \rightarrow B$ can be mapped via a homotopy commutative diagram

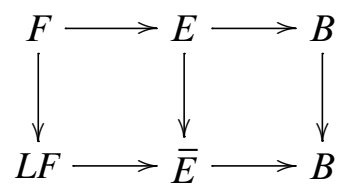

to a fibre sequence over $B$, ie a fibrewise localization exists.

\subsection{Fibrewise localization via homotopy colimit decomposition}

The fibrewise localization for simplicial sets can be defined conveniently using the homotopy colimit decomposition, which can be viewed as a reformulation of the above method for locally trivial morphisms. One writes the fibration $p: E \rightarrow B$ as the map of homotopy colimits hocolim $p^{-1}(\sigma) \rightarrow$ hocolim $\sigma$ over the simplices of the base and defines the fibrewise localization to be the map of homotopy colimits hocolim $L_{f}\left(p^{-1}(\sigma)\right) \rightarrow$ hocolim $\sigma$, cf [5, 1.F.3].

In the simplicial sheaf setting-because the simplices $\sigma: \Delta^{n} \times U \rightarrow B$ are not contractible - we can not simply apply the localization functor. We need to discuss in a little more detail what the localization of the fibre should be. We propose the following definition which, however, only works if the localized model structure is proper. Consider the fibre $p^{-1}(\sigma)$ over the simplex $\sigma: \Delta^{n} \times U \rightarrow B$. We apply 
the localization to this morphism and obtain $L_{f}\left(p^{-1}(\sigma)\right) \rightarrow L_{f}\left(\Delta^{n} \times U\right)$. Now we have localized the fibre, but the base simplex and therefore the whole diagram has changed - the homotopy colimit is not necessarily $B$ any more. Therefore, we factor $L_{f}\left(p^{-1}(\sigma)\right) \rightarrow L_{f}\left(\Delta^{n} \times U\right)$ as a trivial cofibration $L_{f}\left(p^{-1}(\sigma)\right) \rightarrow \widetilde{F}$ and a fibration $\widetilde{F} \rightarrow L_{f}\left(\Delta^{n} \times U\right)$. The pullback $\left(\Delta^{n} \times U\right) \times_{L_{f}\left(\Delta^{n} \times U\right)} \tilde{F}$ is then the best approximation to the localization of $p^{-1}(\sigma)$ which still maps to the (nonlocal) simplex $\Delta^{n} \times U$. Properness of the local model structure is needed to make sure that the morphism

$$
p^{-1}(\sigma) \rightarrow\left(\Delta^{n} \times U\right) \times_{L_{f}\left(\Delta^{n} \times U\right)} \tilde{F}
$$

is an $f$-local weak equivalence. Of course, for a clean definition we need to replace statements and arguments involving "the fibre of $p: E \rightarrow B$ " by corresponding statements about "the diagram of the fibres".

Definition 6.4 Let $T$ be a site, and let $p: E \rightarrow B$ be a morphism of simplicial sheaves on $T$. We consider the category of $(\Delta \times T) \downarrow B$-diagrams in $\Delta^{\text {op }} \mathcal{S} h v(T)$ equipped with the model structure which has the fibrations and weak equivalences from the $f$-local model structure, and cofibrations defined by left lifting property.

From the morphism $p: E \rightarrow B$ we obtain a morphism of diagrams $\mathcal{F} \rightarrow$ id, where

$$
\mathcal{F}: \sigma \mapsto p^{-1}(\sigma), \quad \text { id: }\left(\sigma: \Delta^{n} \times U \rightarrow B\right) \mapsto \Delta^{n} \times U .
$$

We then consider the diagram

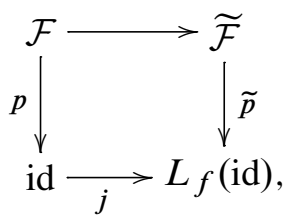

where $j$ is a fibrant replacement in the model structure on the diagram category and $\widetilde{\mathcal{F}}$ is obtained from a factorization of $j \circ p$ as trivial cofibration $\mathcal{F} \rightarrow \widetilde{\mathcal{F}}$ followed by a fibration $\tilde{p}$. The diagram of the " $f$-localized homotopy fibres" is then obtained by the pullback id $\times_{L_{f} \text { (id) }} \widetilde{\mathcal{F}}$. This is a functorial way for assigning to each simplex $\sigma: \Delta^{n} \times U \rightarrow B$ the pullback $\left(\Delta^{n} \times U\right) \times_{L_{f}\left(\Delta^{n} \times U\right)} L_{f}\left(p^{-1}(\sigma)\right)$.

The fibrewise localization $\bar{p}: \bar{E} \rightarrow B$ is then defined to be the homotopy colimit of the diagram id $\times_{L_{f}(\mathrm{id})} \widetilde{\mathcal{F}}$.

Although we have given the definition of fibrewise localization for an arbitrary map, it is only well-behaved for quasifibrations. The following result makes this precise. 
Lemma 6.5 Let $T$ be a site, and $p: E \rightarrow B$ be a sharp map in $\Delta^{\text {op }} \mathcal{S h} v(T)$. Then the fibrewise localization $\bar{p}: \bar{E} \rightarrow B$ is again a sharp map.

Proof Consider a morphism $\phi: \sigma \rightarrow \tau$ of simplices of $B$ over $U$, and complete the following diagram:

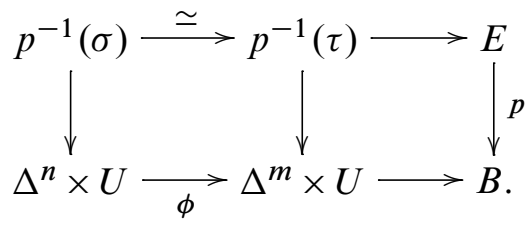

The indicated map $p^{-1}(\sigma) \rightarrow p^{-1}(\tau)$ is a simplicial weak equivalence because $p$ is sharp. Since $f$-localization preserves simplicial weak equivalences, this induces a simplicial weak equivalence $\bar{p}^{-1}(\sigma) \rightarrow \bar{p}^{-1}(\tau)$. A cofibrant replacement of the diagram of " $f$-localized homotopy fibres" as in Definition 6.4 does not change this property. The resulting diagram is a homotopy colimit diagram, hence $\bar{p}^{-1}(\sigma)$ is simplicially weakly equivalent to the homotopy fibre of $\bar{p}$ over $\sigma$. Therefore, the fibrewise localization $\bar{p}: \bar{E} \rightarrow B$ is a sharp map.

This implies that although the definition of fibrewise localization does not necessarily produce a simplicial fibration, it turns sharp maps into sharp maps. The resulting fibrewise localization can then be replaced by an honest simplicial fibration without changing its properties, in particular the weak homotopy type of its fibres. In the following, whenever we use the fibrewise localization, formulations of the results will require the input to fibrewise localization to be a simplicial fibration.

Remark 6.6 (i) In the special case of simplicial sets, where the base can be decomposed into (contractible) simplices, this definition reduces to the usual fibrewise localization. In the simplicial sheaf setting, where the base can not be decomposed into contractible pieces, we use the homotopy fibre definition over the simplices, and the homotopy colimit decomposition to define the fibrewise localization. This construction somehow sits between the "classical" fibrewise localization and the computation of the homotopy fibre-over representable objects we have the homotopy fibre, anything more global than representable objects behaves like fibrewise localization.

(ii) Note finally that if pt denotes the terminal object of $\Delta^{\mathrm{op}} \mathcal{S h v}(T)$, then the induced morphism $p^{-1}(\sigma) \rightarrow \bar{p}^{-1}(\sigma)$ is the $f$-localization for any simplex $\sigma: \Delta^{n} \times \mathrm{pt} \rightarrow B$. 
We now show that our definition of fibrewise localization has the right properties: it is an $f$-local weak equivalence on the total spaces $E \rightarrow \bar{E}$, and on the local fibres it is exactly the "canonical" morphism from point set fibre to " $f$-localized homotopy fibre rel base simplex."

Lemma 6.7 Let $T$ be a site, let $f: X \rightarrow Y$ be a morphism of simplicial sheaves such that the $f$-localized model structure is proper. Let $p: E \rightarrow B$ be a fibration of simplicial sheaves. Then the morphism $E \rightarrow \bar{E}$ from Definition 6.4 is an $f$-local weak equivalence and for each simplex $\sigma: \Delta^{n} \times U \rightarrow B$, the following is an $f$-local homotopy pullback:

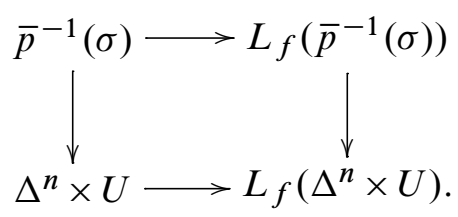

Proof The fact that the diagrams are $f$-local pullbacks for each simplex of the base is a consequence of the definition and properness of the local model structure: by Lemma 3.5, the fibre $\bar{p}^{-1}(\sigma)$ is the space $\left(\Delta^{n} \times U\right) \times{ }_{L_{f}\left(\Delta^{n} \times U\right)} L_{f}\left(p^{-1}(\sigma)\right)$ which is defined as the pullback

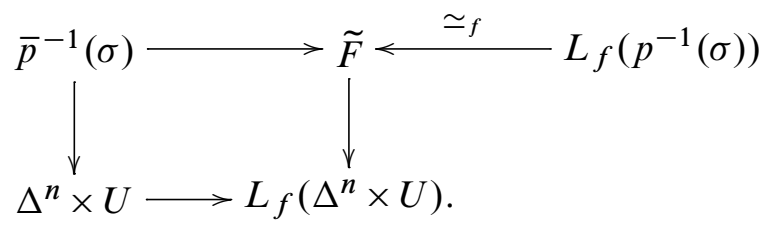

In particular, by properness of the local model structure $\bar{p}^{-1}(\sigma) \rightarrow \widetilde{F}$ is an $f$-local weak equivalence and $\widetilde{F}$ is $f$-local by definition. The above pullback is obviously an $f$-local homotopy pullback, and it is simplicially equivalent to the one claimed in the diagram.

Now consider the commutative diagram

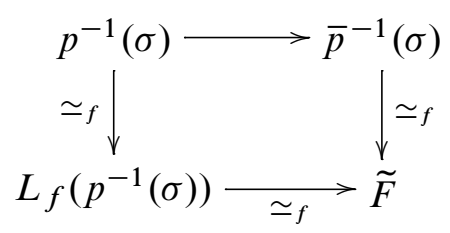

arising from the definition of $\bar{p}^{-1}(\sigma)$. The left and bottom morphism are $f$-local weak equivalences by construction. We saw above that the right morphism is also an $f$-local weak equivalence by properness. Therefore, the top morphism is an $f$-local 
weak equivalence. The morphism $E \rightarrow \bar{E}$ is the homotopy colimit of the morphisms $p^{-1}(\sigma) \rightarrow \bar{p}^{-1}(\sigma)$, and is therefore an $f$-local weak equivalence.

The properties established by the lemma above could be seen as an adequate definition of fibrewise localization in the simplicial sheaf setting-an $f$-local equivalence on the total space and a suitable localization morphism on the fibres. The lemma also allows us to formulate what it means for a map to have " $f$-local fibres".

Definition 6.8 Under the conditions of Lemma 6.7, a simplicial fibration $p: E \rightarrow B$ is said to have $f$-local fibres if one of the following equivalent definitions is satisfied:

(i) the morphism $E \rightarrow \bar{E}$ is a simplicial weak equivalence;

(ii) for any simplex $\sigma: \Delta^{n} \times U \rightarrow B$ the following induced commutative diagram is a simplicial homotopy pullback:

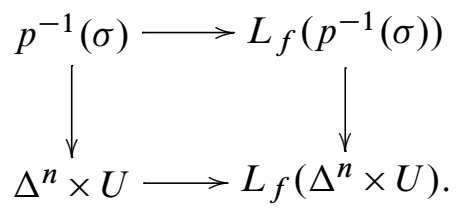

Lemma 6.9 Assume the conditions of Lemma 6.7, let $p: E \rightarrow B$ be a simplicial fibration in $\Delta^{\mathrm{op}} \operatorname{Shv}(T)$ and let $\bar{p}: \bar{E} \rightarrow B$ be its fibrewise localization. Then $p$ is universally $f$-local if and only if $\bar{p}$ is.

Proof It follows from Lemma 6.7 that the following two pullbacks are $f$-locally weakly equivalent:
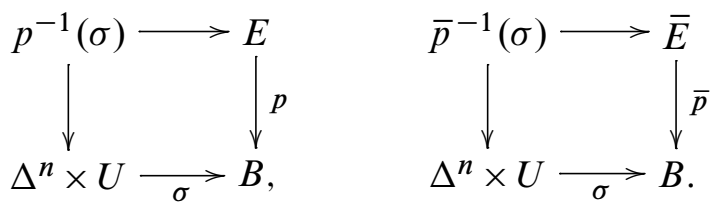

This implies the claim.

\subsection{Comparison results}

Lemma 6.10 Let $T$ be a site, let $f: X \rightarrow Y$ be a morphism of simplicial sheaves on $T$ such that the $f$-local model structure is proper, and let $p: E \rightarrow B$ be a fibration of simplicial sheaves. 
(i) Assume there exists a base point $\mathrm{pt} \rightarrow B$. There exists a morphism $\bar{E}^{C S} \rightarrow \bar{E}^{H D}$ from the Chataur-Scherer fibrewise localization to the fibrewise localization defined using the homotopy colimit decomposition. This morphism induces simplicial weak equivalences over simplices $\Delta^{n} \times \mathrm{pt} \rightarrow B$ over the chosen base point $\mathrm{pt} \rightarrow B$.

(ii) If $p: E \rightarrow B$ is locally trivial, there exists a morphism $\bar{E}^{B} \rightarrow \bar{E}^{H D}$ from the fibrewise localization using the classifying space to the fibrewise localization using the homotopy colimit decomposition. This morphism is a simplicial weak equivalence.

Proof We only sketch (i). The Chataur-Scherer fibrewise localization is constructed as a transfinite telescope in which the successor steps are given by the following diagram:

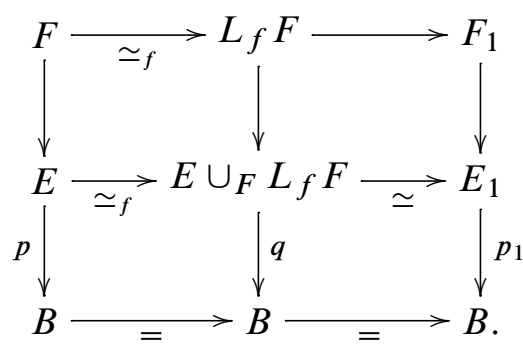

One starts with the fibre sequence $F=p^{-1}(\sigma) \rightarrow E \rightarrow B$, takes the localization $F \rightarrow L_{f} F$ and then the pushout. The resulting middle column is not a fibre sequence, so we replace $q$ by a fibration $p_{1}$, and let $F_{1} \rightarrow E_{1} \rightarrow B$ be the new fibre sequence. It is then easy to see that the morphism $E \rightarrow \bar{E}^{H D}$ factors through $E_{1} \rightarrow \bar{E}^{H D}$. This implies the existence of the required morphism. For any simplex $\Delta^{n} \times \mathrm{pt} \rightarrow B$, we have an induced composition

$$
F \rightarrow\left(\bar{p}^{C S}\right)^{-1}(\sigma) \rightarrow\left(\bar{p}^{H D}\right)^{-1}(\sigma),
$$

where the first morphism and the composition are both $f$-localizations of $F$. The second morphism then must be a simplicial weak equivalence as claimed.

(ii) We apply the homotopy colimit decomposition construction of the fibrewise localization to the universal locally trivial fibration $B(*$, hAut $(F), F) \rightarrow B$ hAut $(F)$. Using properness, it can be checked that the result is a locally trivial fibre sequence with fibre $L_{f} F$. The induced morphism $B$ hAut॰ $(F) \rightarrow B$ hAut $\left(L_{f} F\right)$ is the localization morphism, because locally (over simplices where $p: E \rightarrow B$ is trivial) the induced morphism is the localization morphism. This means that both methods of fibrewise localization agree on the universal object, so they agree. 


\section{Characterization of universally $f$-local maps}

Lemma 7.1 A simplicial fibration $p: E \rightarrow B$ with $f$-local fibres in the sense of Definition 6.8 is universally $f$-local if and only if the following square is a simplicial homotopy pullback:

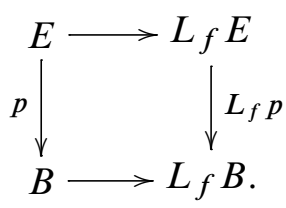

Proof Assume that the square is a simplicial homotopy pullback. Then up to simplicial weak equivalence, the map $p: E \rightarrow B$ is the pullback of $L_{f} E \rightarrow L_{f} B$, which is universally $f$-local by Corollary 5.6. By Corollary 5.4 , it is universally $f$-local. Note that the map $p: E \rightarrow B$ then automatically has $f$-local fibres, by applying the homotopy pullback lemma to the following cube:

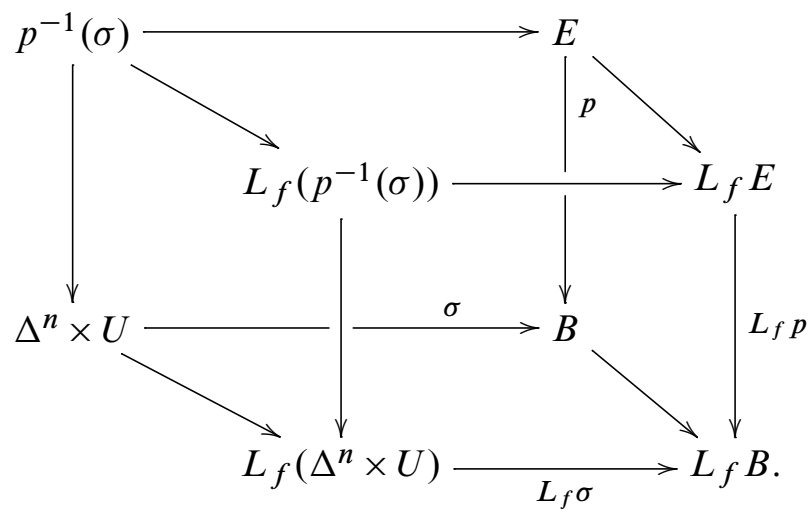

Now assume that $p: E \rightarrow B$ is universally $f$-local with $f$-local fibres. Consider the diagram

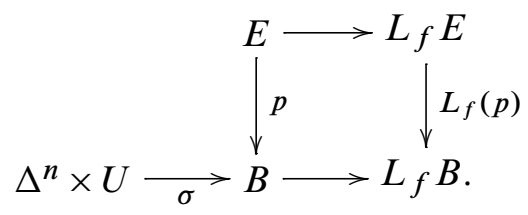

We can assume that $L_{f}(p): L_{f} E \rightarrow L_{f} B$ is a simplicial fibration. To check that the square is a simplicial homotopy pullback, it suffices to show that for each simplex $\sigma$ as above the induced morphism of fibres $p^{-1}(\sigma) \rightarrow\left(L_{f} p\right)^{-1}(\sigma)$ is a simplicial weak equivalence. This morphism is an $f$-local weak equivalence because $p$ and $L_{f}(p)$ 
are universally $f$-local.

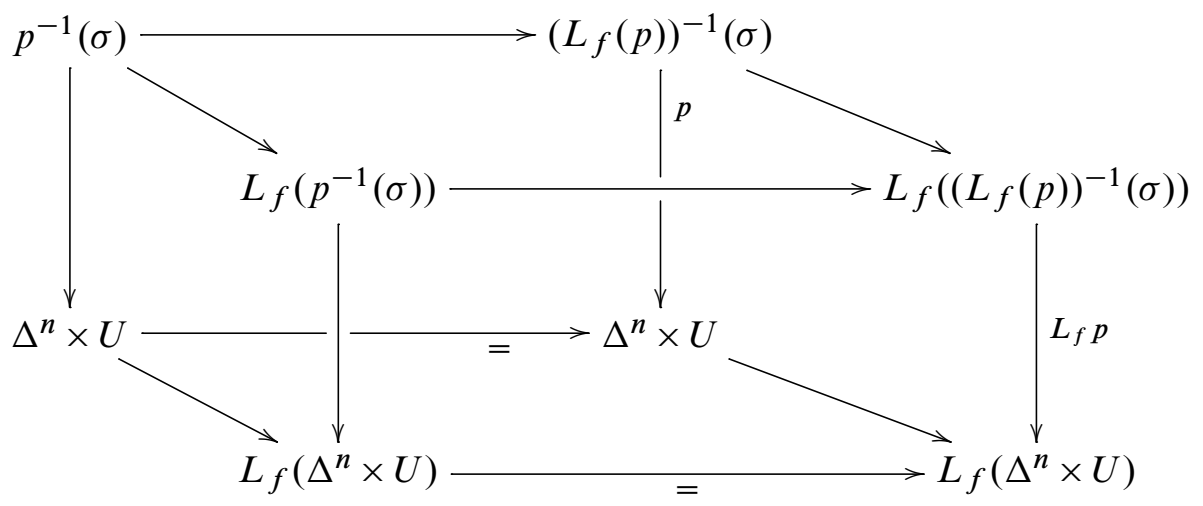

The front square is a simplicial homotopy pullback because its top morphism

$$
L_{f}\left(p^{-1}(\sigma)\right) \rightarrow L_{f}\left(\left(L_{f}(p)\right)^{-1}(\sigma)\right)
$$

is the localization of an $f$-local weak equivalence, hence a simplicial weak equivalence. The side squares are both simplicial homotopy pullbacks because both maps $p$ and $L_{f}(p)$ have $f$-local fibres. The back square is thus a simplicial homotopy pullback, so the morphism in question is a simplicial weak equivalence.

The following plays the role of [1, Lemma 3.2].

Lemma 7.2 Let $p: E \rightarrow B$ and $j: B \rightarrow C$ be simplicial fibrations of simplicial sheaves with $f$-local $C$. Then $p: E \rightarrow B$ is universally $f$-local if and only if for each simplex $\sigma: \Delta^{n} \times U \rightarrow C$ the induced map $\sigma^{*}(p):(j \circ p)^{-1}(\sigma) \rightarrow j^{-1}(\sigma)$ is universally $f$-local.

Proof By Corollary 5.4, only the "if"-direction needs proof here.

Assume $\tilde{\sigma}: \Delta^{n} \times U \rightarrow B$ is a simplex of $B$. Set $\sigma=j \circ \tilde{\sigma}$ and consider the diagram

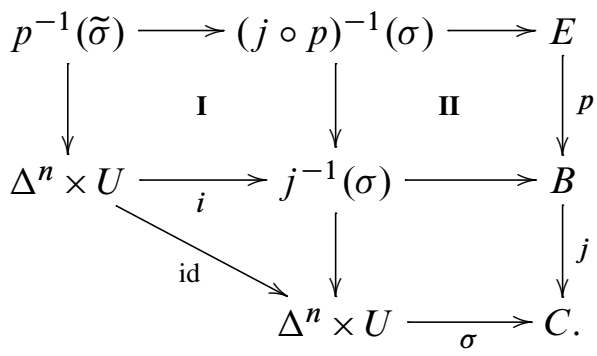


By assumption, the morphism $\sigma^{*}(p):(j \circ p)^{-1}(\sigma) \rightarrow j^{-1}(\sigma)$ is universally $f$-local, so the square $\mathbf{I}$ is an $f$-local homotopy pullback. Therefore, $p$ is universally $f$-local if $\mathbf{I I}$ is an $f$-local homotopy pullback, because then the composition $\mathbf{I}+\mathbf{I I}$ is an $f$-local homotopy pullback.

To see the latter, we consider the following part of the previous diagram:

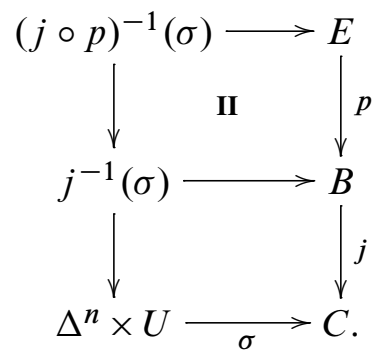

By Corollary 5.6, both $j$ and $j \circ p$ are universally $f$-local. In particular, the outer and the lower square are $f$-local homotopy pullbacks, so the upper square is an $f$-local homotopy pullback.

Theorem 7.3 Let $T$ be a site and let $f: X \rightarrow Y$ be a morphism of simplicial sheaves in $\Delta^{\mathrm{op}} \mathcal{S h v}(T)$. Assume that the $f$-local model structure is proper. Let $p: E \rightarrow B$ be a simplicial fibration of simplicial sheaves.

We denote by $\bar{p}: \bar{E} \rightarrow B$ the fibrewise $f$-localization of $p$, and by $j: B \rightarrow L_{f} B$ an $f$-local fibrant replacement of $B$. The following are equivalent, where (iv) only makes sense if $p: E \rightarrow B$ is locally trivial:

(i) the map $p: E \rightarrow B$ is universally $f$-local;

(ii) the fibrewise localization $\bar{p}: \bar{E} \rightarrow B$ is universally $f$-local;

(iii) for each simplex $\sigma: \Delta^{n} \times U \rightarrow L_{f} B$, the following canonical diagram is a simplicial homotopy pullback:

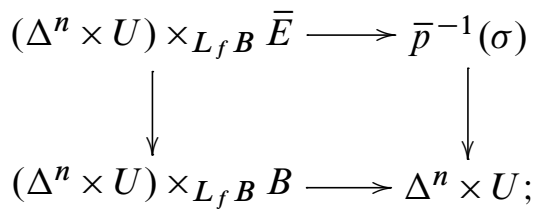

(iv) for each simplex $\sigma: \Delta^{n} \times U \rightarrow L_{f} B$, the composition

$$
\left(\Delta^{n} \times U\right) \times_{L_{f} B} B=j^{-1}(\sigma) \rightarrow B \rightarrow B \text { hAut॰ }(F) \rightarrow B \text { hAut॰ }\left(L_{f} F\right)
$$

factors through the projection $\left(\Delta^{n} \times U\right) \times{ }_{L_{f} B} B \rightarrow \Delta^{n} \times U$ (in the simplicial homotopy category). 
Proof Note that by a simplicial fibrant replacement, we can and do assume that $j: B \rightarrow L_{f} B$ is a simplicial fibration.

We first prove the equivalence between (i) and (ii). Let $p: E \rightarrow B$ be universally $f$-local. Consider the diagram

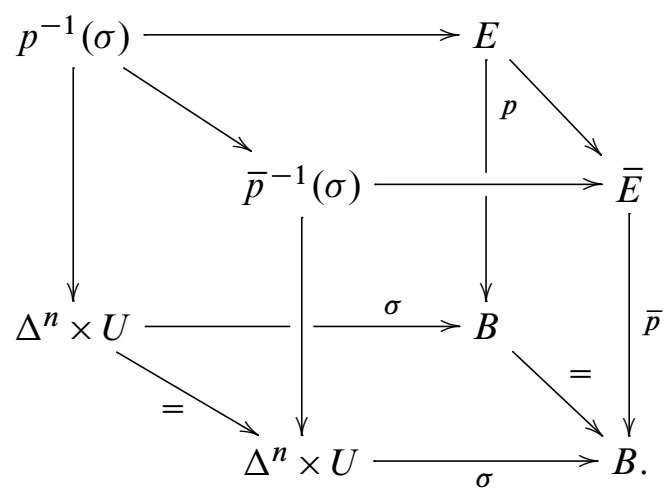

By definition of fibrewise localization and Lemma 6.7, the morphisms $E \rightarrow \bar{E}$ and $p^{-1}(\sigma) \rightarrow \bar{p}^{-1}(\sigma)$ are $f$-local weak equivalences. Therefore, the front square is an $f$-local homotopy pullback if and only if the back square is an $f$-local homotopy pullback. This proves the equivalence of (i) and (ii).

Assume that $p: E \rightarrow B$ is locally trivial with fibre $F$ in the sense of [18, Definition 3.5], in particular $p$ is classified by a morphism $B \rightarrow B$ hAut. $(F)$. By Lemma 6.10, the fibrewise localization can be described as the pullback of the universal $L_{f} F$-fibration along the composition $B \rightarrow B$ hAut॰ $(F) \rightarrow B$ hAut $\left(L_{f} F\right)$. The fact that the canonical diagram in (iii) is a simplicial homotopy pullback is then simply a reformulation of the fact that the composition in (iv) factors through the projection $j^{-1}(\sigma) \rightarrow \Delta^{n} \times U$. Therefore, (iii) and (iv) are also equivalent provided (iv) makes sense.

It remains to prove the equivalence of (ii) and (iii). We complete the diagram in (iii) as follows:

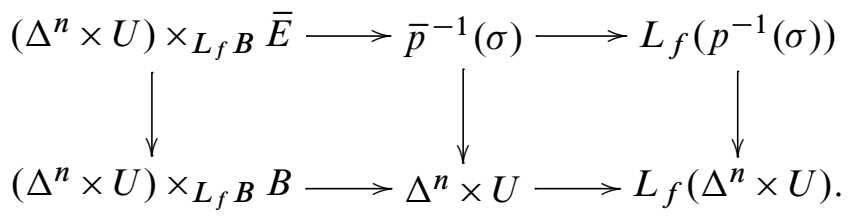

The right square is a simplicial homotopy pullback by the definition of fibrewise localization, cf Lemma 6.7. The right vertical map is simplicially equivalent to the localization of the left vertical map. Therefore, by Lemma 7.1, the outer square is a simplicial homotopy pullback if and only if the left vertical map is universally $f$-local. The left 
square is a simplicial homotopy pullback if and only if (ii) holds. For the equivalence of (ii) and (iii) it then suffices to show that $\bar{p}: \bar{E} \rightarrow B$ is universally $f$-local if and only if for each simplex $\sigma: \Delta^{n} \times U \rightarrow L_{f} B$, the induced map $(j \circ \bar{p})^{-1}(\sigma) \rightarrow j^{-1}(\sigma)$ is universally $f$-local. This is the statement of Lemma 7.2.

Remark 7.4 The above result can probably not be effectively used for showing that a given map is universally $f$-local. However, it explains philosophically why a map can fail to be universally $f$-local. In spite of added complication of considering all the local fibres of $p$, the reason is still the same as in [1]: a map fails to be universally $f$-local if its fibrewise localization is nontrivial over nonlocal parts. In the simplicial situation, one pulls back the fibrewise localization to the fibre $A_{L_{f} B}$ of $j: B \rightarrow L_{f} B$. In the sheaf situation, one has to replace the single space $A_{L_{f} B}$ by the set of all the fibres of $B \rightarrow L_{f} B$ over the various simplices. Note that the above result reduces exactly to [1, Theorem 4.1$]$ for $T=$ pt.

\section{Application: fibrations in $\mathbb{A}^{1}$-homotopy theory}

In this section, we apply the localization theory developed earlier to discuss fibrations in $\mathbb{A}^{1}$-homotopy theory. Hence we specialize to the site $T=\mathrm{Sm}_{k}$ of smooth finite type schemes over a field $k$ equipped with the Zariski or Nisnevich topology. We consider the injective model structure on the category of simplicial sheaves $\Delta^{\mathrm{op}} \mathcal{S} h v\left(\operatorname{Sm}_{k}\right)$, and apply a Bousfield localization to the scheme $\mathbb{A}^{1}$ considered as constant representable simplicial sheaf. More details on the construction of $\mathbb{A}^{1}$-homotopy theory can be found in [14].

Now recall from [18], that for each simplicial sheaf $F$, there is a classifying space of locally trivial maps with fibre $F$ in the sense of [18, Definition 3.5]. We denote this space by $B$ hAut. $(F)$, since [18, Theorem 5.10] shows this space can be constructed as the classifying space of the simplicial sheaf of monoids hAut. $(F)$ of homotopy self-equivalences of $F$. We assume here the morphisms considered are locally trivial in the Nisnevich topology. Note in the above, we are working in the unpointed category, so we can not talk about fibre sequences in the sense of [10, Definition 6.2.6]. Also the classification result cited is a classification in the unpointed setting.

The main general result is the following.

Theorem 8.1 Let $X$ be a cofibrant and $\mathbb{A}^{1}$-local fibrant simplicial sheaf on $\mathrm{Sm}_{k}$. Then $B$ hAut. $(X)$ is $\mathbb{A}^{1}$-local if and only if the sheaf of homotopy self-equivalence groups $\pi_{0}$ (hAut. $(X)$ ) is strongly $\mathbb{A}^{1}$-invariant. 
Proof We first prove the simplicial sheaf of monoids of homotopy self-equivalences hAut. $(X)$ is fibrant and $\mathbb{A}^{1}$-local.

By [14, Lemma I.1.8], there is a fibration

$$
\operatorname{Hom}(X, Y) \rightarrow Y
$$

if $Y$ is fibrant. Thus $\operatorname{Hom}(X, X)$ is fibrant if $X$ is fibrant. The simplicial set hAut. $(X)(U)$ is a union of connected components of $\operatorname{Hom}(X, X)(U)$. By 2 out of 3 for weak equivalences a morphism $f: X \times U \rightarrow X \times U$ is a weak equivalence if it is homotopic to a morphism $f^{\prime}: X \times U \rightarrow X \times U$ which is a weak equivalence. Therefore hAut. $(X)(U)$ consists exactly of the union of the components of $\operatorname{Hom}(X, X)(U)$ which contain weak equivalences.

Consider now the commutative diagram

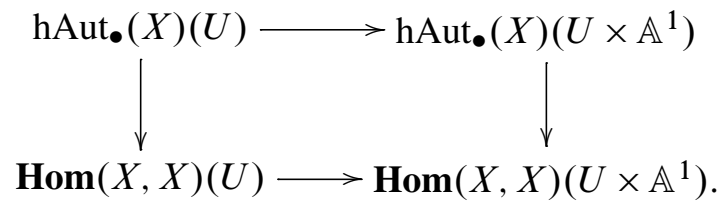

The vertical arrows are the inclusions as described above, and the lower horizontal morphism is a weak equivalence of simplicial sets since we noted that $\operatorname{Hom}(X, X)$ is $\mathbb{A}^{1}$-local. In particular, the lower morphism induces a bijection on the connected components. This bijection restricts to a bijection between the components consisting of weak equivalences: first, any morphism $f: X \times U \rightarrow X \times U$ is a retract of the morphism $f \times$ id: $X \times U \times \mathbb{A}^{1} \rightarrow X \times U \times \mathbb{A}^{1}$, therefore the preimage of a component in hAut॰ $(X)\left(U \times \mathbb{A}^{1}\right)$ is in hAut• $(X)(U)$. Similarly, if $f$ is a weak equivalence, then $f \times$ id is a weak equivalence. But then the morphism hAut. $_{\bullet}(X)(U) \rightarrow$ hAut $_{\bullet}(X)\left(U \times \mathbb{A}^{1}\right)$ is a weak equivalence because it is a bijection on connected components, and the connected components are connected components of the mapping spaces $\operatorname{Hom}(X, X)$, where we have a weak equivalence. This implies that hAut॰ $(X)$ is $\mathbb{A}^{1}$-local if $X$ is $\mathbb{A}^{1}$-local.

By Morel [13, Lemma 6.45, Theorem 6.46], $B$ hAut॰ $(X)$ is $\mathbb{A}^{1}$-local if and only if the sheaf of groups $\pi_{0} L_{\mathbb{A}^{1}} \Omega B$ hAut• $X$ is strongly $\mathbb{A}^{1}$-invariant. The theorem follows, if we can prove that the obvious morphism

$$
\text { hAut. } X \rightarrow \Omega B \text { hAut॰ } X \rightarrow L_{\mathbb{A}^{1}} \Omega B \text { hAut• } X
$$

induces an isomorphism of sheaves of groups $\pi_{0}$. But the obvious morphism

$$
\text { hAut• } X \rightarrow \Omega B \text { hAut } X
$$


is already a weak equivalence of simplicial sheaves, because the stalks of hAut. $X$ are monoids of homotopy self-equivalences of simplicial sets which are group-like. Thus, the morphism induces weak equivalences on the stalks, cf Rudyak [16, Corollary IV.1.68]. This implies that $\Omega B$ hAut॰ $X$ is already $\mathbb{A}^{1}$-local, hence the localization $\Omega B$ hAut॰ $X \rightarrow L_{\mathbb{A}^{1}} \Omega B$ hAut॰ $X$ is a simplicial weak equivalence.

This result has the following consequence. Note that in the following, we are talking about locally trivial maps, so the fibrewise localization can be defined on classifying spaces. Note also that the statement "the map $p: E \rightarrow B$ is universally $\mathbb{A}^{1}$-local" implies that for any choice of base point $x$ : Spec $k \rightarrow B$, the resulting sequence $p^{-1}(x) \rightarrow E \rightarrow B$ is a fibre sequence with $p^{-1}(x) \simeq_{\mathbb{A}^{1}} F$.

Corollary 8.2 Let $X$ be a cofibrant, fibrant and $\mathbb{A}^{1}$-local simplicial sheaf on $\mathrm{Sm}_{k}$ such that $\pi_{0}$ (hAut. $\left.(X)\right)$ is strongly $\mathbb{A}^{1}$-invariant.

Then we have the following statements:

(i) the morphism

$$
B(*, \text { hAut॰ } X, X) \rightarrow B \text { hAut॰ } X
$$

is universally $\mathbb{A}^{1}$-local;

(ii) any Nisnevich locally trivial morphism $E \rightarrow B$ whose fibre $F$ has the $\mathbb{A}^{1}$ homotopy type of $X$ is also universally $\mathbb{A}^{1}$-local;

(iii) denoting by $\mathcal{H}^{\mathbb{A}^{1}}(Y, X)$ the pointed set of Nisnevich locally trivial fibre sequences over $Y$ with fibre $X$ up to $\mathbb{A}^{1}$-equivalence, we have a natural bijection

$$
\mathcal{H}^{\mathbb{A}^{1}}(-, X) \cong[-, B \text { hAut. } X]_{\mathbb{A}^{1}} .
$$

Proof (i) Corollary 5.6 implies that the universal fibre sequence is $\mathbb{A}^{1}$-local if (a simplicial fibrant replacement of) the classifying space $B$ hAut. $X$ is $\mathbb{A}^{1}$-local. But $B$ hAut. $X$ is $\mathbb{A}^{1}$-local since the conditions of Theorem 8.1 are satisfied.

Part (ii) follows from Corollary 5.4. Any Nisnevich locally trivial fibre sequence is a pullback of the universal fibre sequence with fibre $F$ along some morphism $B \rightarrow B$ hAut. $F$. But from (i) it follows that the universal fibre sequence over $B$ hAut. $L_{\mathbb{A}^{1}} F \simeq B$ hAut॰ $X$ is universally $\mathbb{A}^{1}$-local.

For (iii) we first note that [18, Theorem 5.10] yields a bijection

$$
\mathcal{H}(-, X) \cong[-, B \text { hAut• } X]
$$


For the definition of $\mathcal{H}$, cf [18, Definition 5.1]. Since $B$ hAut. $X$ is $\mathbb{A}^{1}$-local, we also have a bijection

$$
[-, B \text { hAut. } X] \cong[-, B \text { hAut. } X]_{\mathbb{A}^{1}} .
$$

Conversely, since $B$ hAut॰ $X$ is $\mathbb{A}^{1}$-local, the classifying morphism $B \rightarrow B$ hAut॰ $X$ factors up to homotopy through a morphism $L_{\mathbb{A}^{1}} B \rightarrow B$ hAut。 $X$. By Corollary 5.6, we can hence assume that the fibre sequence classified by this consists of $\mathbb{A}^{1}$-local spaces. Since a morphism between local spaces is an $\mathbb{A}^{1}$-weak equivalence if and only if it is a simplicial weak equivalence, the two equivalence notions for fibre sequences coincide, and we have the final bijection $\mathcal{H}^{\mathbb{A}^{1}}(-, X) \cong \mathcal{H}(-, X)$.

Remark 8.3 Weaker versions of the above have been used in [13] and by the author in [19] to produce fibre sequences from torsors under algebraic groups. The above statement can be used to produce classifying spaces for many other "fibre sequences" in $\mathbb{A}^{1}$-homotopy theory where the structure groups are no longer algebraic groups. One particularly interesting such classifying space would be the classifying space of spherical fibrations: let $S^{2 n, n}=S^{n} \wedge \mathbb{G}_{m}^{\wedge n}$ be an $\mathbb{A}^{1}$-local model of the $(2 n, n)$-sphere. Then the Nisnevich locally trivial morphisms of simplicial sheaves with fibre $S^{2 n, n}$ are classified by $B$ hAut. $S^{2 n, n}$. This remains true in the $\mathbb{A}^{1}$-local situation if the sheaf of homotopy self-equivalences $\pi_{0}$ hAut॰ $S^{2 n, n}$ of the $(2 n, n)$-sphere is strongly $\mathbb{A}^{1}$-invariant. By the computations in [13, Corollary 6.43, Theorem 7.36 resp. Corollary 7.40], the homotopy endomorphisms of $S^{2 n, n}$ are given by the Grothendieck-Witt ring $G W(k)$ for $n \geq 2$ and an extension of $G W(k)$ for $n=1$. The homotopy self-equivalences are then the units in the above rings.

If the sheaf of units of the Grothendieck-Witt sheaf $G W$ are strongly $\mathbb{A}^{1}$-invariant, then $B$ hAut. $S^{2 n, n}$ is an $\mathbb{A}^{1}$-local classifying space for spherical fibrations. Unconditionally, its universal $\mathbb{A}^{1}$-covering-the classifying space of the connected component of hAut. $S^{2 n, n}$-is an $\mathbb{A}^{1}$-local classifying space for orientable spherical fibrations.

There are several interesting directions to pursue here:

(i) How does the notion of orientability coming from spherical fibrations relate to other notions of orientability in $\mathbb{A}^{1}$-homotopy theory?

(ii) I would expect that the classifying space for orientable spherical fibrations is cellular with a cell structure similar to the one known in "classical algebraic topology". This would imply that the characteristic classes of orientable spherical fibrations over an algebraically closed field coincide with the known topological characteristic classes. 
(iii) There is an obvious morphism $B G L_{n} \rightarrow B$ hAut. $S^{2 n, n}$ obtained from a change of fibre along $\mathbb{A}^{n} \rightarrow \mathbb{A}^{n} /\left(\mathbb{A}^{n} \backslash\{0\}\right) \simeq S^{2 n, n}$-the classifying space version of the $J$-homomorphism. This could possibly be used in connection with the characteristic classes in (ii) to exhibit simplicial sheaves with a reasonably behaved spherical fibration which are not $\mathbb{A}^{1}$-weakly equivalent to any smooth projective scheme.

Finally, I would like to remark that an $f$-local version of homotopy distributivity does not hold: a homotopy colimit of universally $f$-local maps is not necessarily universally $f$-local. In particular, it is not necessarily true that a map which is locally trivial is universally $f$-local. As an example, let $G$ be a sheaf of groups on $\operatorname{Sm}_{k}$ which is $\mathbb{A}^{1}$-invariant but not strongly $\mathbb{A}^{1}$-invariant. Then the map $E G \rightarrow B G$ is not universally $f$-local-its simplicial homotopy fibre is $G$ (which is $\mathbb{A}^{1}$-local by assumption), but its $\mathbb{A}^{1}$-homotopy fibre is $\pi_{1}\left(L_{\mathbb{A}^{1}} B G\right)$. If $\pi_{1} B G \cong \pi_{1}\left(L_{\mathbb{A}^{1}} B G\right)$, then $G$ would be strongly $\mathbb{A}^{1}$-invariant, contradicting the assumption. In particular, it seems that the condition on strong $\mathbb{A}^{1}$-invariance of self-equivalences in Corollary 8.2 can not be dropped.

\section{References}

[1] A J Berrick, E D Farjoun, Fibrations and nullifications, Israel J. Math. 135 (2003) 205-220 MR1997044

[2] D Chataur, J Scherer, Fiberwise localization and the cube theorem, Comment. Math. Helv. 81 (2006) 171-189 MR2208803

[3] A Dold, R Thom, Quasifaserungen und unendliche symmetrische Produkte, Ann. of Math. 67 (1958) 239-281 MR0097062

[4] D Dugger, Combinatorial model categories have presentations, Adv. Math. 164 (2001) 177-201 MR1870516

[5] E D Farjoun, Cellular spaces, null spaces and homotopy localization, Lecture Notes in Mathematics 1622, Springer, Berlin (1996) MR1392221

[6] P G Goerss, J F Jardine, Localization theories for simplicial presheaves, Canad. J. Math. 50 (1998) 1048-1089 MR1650938

[7] P G Goerss, J F Jardine, Simplicial homotopy theory, Progress in Mathematics 174, Birkhäuser, Basel (1999) MR1711612

[8] PS Hirschhorn, Model categories and their localizations, Mathematical Surveys and Monographs 99, American Mathematical Society (2003) MR1944041

[9] J Hornbostel, Localizations in motivic homotopy theory, Math. Proc. Cambridge Philos. Soc. 140 (2006) 95-114 MR2197578 
[10] M Hovey, Model categories, Mathematical Surveys and Monographs 63, American Mathematical Society (1999) MR1650134

[11] J F Jardine, Boolean localization, in practice, Doc. Math. 1 (1996) No. 13, 245-275 MR1405671

[12] J F Jardine, Motivic symmetric spectra, Doc. Math. 5 (2000) 445-553 MR1787949

[13] F Morel, $\mathbb{A}^{1}$-algebraic topology over a field, Lecture Notes in Mathematics 2052, Springer, Heidelberg (2012) MR2934577

[14] F Morel, V Voevodsky, $\mathbf{A}^{1}$-homotopy theory of schemes, Inst. Hautes Études Sci. Publ. Math. (1999) 45-143 MR1813224

[15] C Rezk, Fibrations and homotopy colimits of simplicial sheaves arXiv: math/9811038

[16] Y B Rudyak, On Thom spectra, orientability, and cobordism, Springer Monographs in Mathematics, Springer, Berlin (1998) MR1627486

[17] M Wendt, On fibre sequences in motivic homotopy theory, $\mathrm{PhD}$ thesis, Universität Leipzig (2007)

[18] M Wendt, Classifying spaces and fibrations of simplicial sheaves, J. Homotopy Relat. Struct. 6 (2011) 1-38 MR2818697

[19] M Wendt, Rationally trivial torsors in $\mathbb{A}^{1}$-homotopy theory, J. K-Theory 7 (2011) 541-572 MR2811715

Mathematisches Institut, Albert-Ludwigs-Universität Freiburg

Eckerstraße 1, D-79104, Freiburg im Breisgau, Germany

matthias.wendt@math.uni-freiburg.de

Received: 25 April 2012 Revised: 6 February 2013 